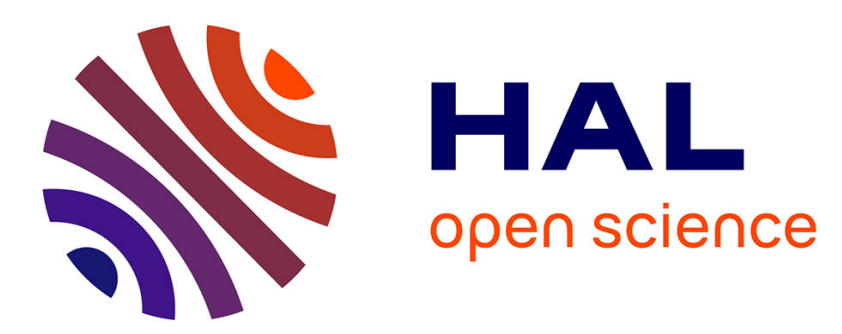

\title{
Influence du nombre de pièges à eau colorés en jaune et de la distance entre ces pièges sur les captures d'aphides
}

Jean-Michel Rabasse, Etienne Brunel, Joëlle Rouzé-Jouan

\section{To cite this version:}

Jean-Michel Rabasse, Etienne Brunel, Joëlle Rouzé-Jouan. Influence du nombre de pièges à eau colorés en jaune et de la distance entre ces pièges sur les captures d'aphides. Agronomie, 1982, 2 (7), pp.647-653. hal-00884429

\section{HAL Id: hal-00884429 \\ https://hal.science/hal-00884429}

Submitted on 1 Jan 1982

HAL is a multi-disciplinary open access archive for the deposit and dissemination of scientific research documents, whether they are published or not. The documents may come from teaching and research institutions in France or abroad, or from public or private research centers.
L'archive ouverte pluridisciplinaire HAL, est destinée au dépôt et à la diffusion de documents scientifiques de niveau recherche, publiés ou non, émanant des établissements d'enseignement et de recherche français ou étrangers, des laboratoires publics ou privés. 


\title{
Influence du nombre de pièges à eau colorés en jaune et de la distance entre ces pièges sur les captures d'aphides
}

\author{
Jean-Michel RABASSE, Etienne BRUNEL $\left({ }^{*}\right)$ \& Joëlle ROUZÉ-JOUAN $\left({ }^{*}\right)$
}

I.N.R.A., Station de Zoologie et de Lutte biologique, 37, boulevard du Cap, F 06602 Antibes. $\left(^{*}\right)$ I.N.R.A., Laboratoire de Recherches de la Chaire de Zoologie de l'E.N.S.A., F 35650 Le Rheu.

\section{RÉSUMÉ}

Piège coloré, Méthodologie, Aphides, Cavariella aegopodii.
Ce travail porte sur les variations des captures de pucerons dans des dispositifs comportant de nombreux pièges jaunes séparés par des distances variées. L'essai a eu lieu dans un champ de carottes, aussi les captures de Cavariella aegopodii Scop., particulièrement abondant, ont-elles été spécialement étudiées. Des hétérogénéités dans les captures ont été mises en évidence ; dans certains cas seulement, clles sont facilement explicables par la proximité de haies. Le nombre de captures par piège diminue lorsque la distance entre pièges augmente, puis il se stabilise, suggérant qu'à l'« effet piège » s'ajoute un « effet dispositif ». Cette même constatation a été faite en ce qui concerne l'indice de diversité des captures de Williams, qui est nettement plus élevé lorsque les pièges sont rapprochés. Enfin, le coefficient de variation des captures de C. aegopodii est dans cet essai de 19 à 34 p. 100, ce qui permet de schématiser la relation entre nombre de pièges et précision et de tirer un certain nombre de conclusions sur la taille du dispositif à utiliser.

\section{SUMMARY}

Yellow water traps Methodology,

Aphids,

Cavariclla aegopodii.

\begin{abstract}
Number of yellow water traps and distance between them in relation to the structure of aphid catches
Yellow water traps are commonly used for collecting populations of flying aphids. This note deals with the influence of the number of small traps ( 10 and $15 \mathrm{~cm}$ in diameter) and of the distance between them (from 2 to $8 \mathrm{~m}$ ) on the catches. Several sets of traps were arranged (fig. 1) in a carrot field, where Cavariella aegopodii Scop. was the outstanding species. Heterogeneity was found in the catches in each set of traps (fig. 1 ; table 1) ; only part of which could be easily explained by the proximity of hedgerows. As the distance between traps increases, the number of carrot-willow aphids caught is first reduced, then becomes stable (tables 2 and 3). For that reason, the authors suggest that attraction to one trap is modified by the attraction of the set of traps. In the same way, the WILlIAMS diversity index is significantly higher when the traps are near to each other (table 4). Finally, the coefficient of variation of the catches of C. aegopodii lies between $19 \%$ and $34 \%$, which, according to the data of figure 2 , allows one to decide the number of traps needed.
\end{abstract}

\section{INTRODUCTION}

Ce travail constitue le $3^{\mathrm{c}}$ volet de l'étude méthodologique des captures d'aphides dans les pièges à eau colorés en jaune. Après avoir étudié l'incidence des caractéristiques du piège lui-même : hauteur (ROBERT et al., 1974 ; BRUNEL et al., 1980), forme et taille (RABASSE et al., 1976) sur les captures, nous prenons maintenant en compte un groupe de pièges en observant les effets du nombre de pièges, de la distance entre ceux-ci et de la localisation du dispositif de piégeage sur le résultat.

\section{MATÉRIEL ET MÉTHODES}

Cet essai s'est déroulé à Bas Courtil (Manche) dans une grande parcelle (2,5 ha environ) de très jeunes carottes au stade 8 feuilles vraies. Cette parcelle était partiellement entourée de haies et jouxtait un champ de maïs (fig. 1). Le piégeage a duré du 15 au 18 mai 1974, période pendant laquelle le temps était beau et ensoleillé, favorable au vol des aphides (températures maximale : $20,8^{\circ} \mathrm{C}$, minimale : $8,6^{\circ} \mathrm{C}$; pas de pluie; $75 \mathrm{~h}$ d'ensoleillement dans la semaine).

L'essai comportait des pièges circulaires de 2 tailles: $10 \mathrm{~cm}$ et $15 \mathrm{~cm}$ de diamètre. Ces pièges étaient peints intérieurement en jaune «bouton d'or» (caractéristiques spectrales précisées par BRUNEL \& LANGOUET, 1970). Ils contenaient de l'eau additionnée de mouillant et étaient disposés sur le sol. Les pièges étaient regroupés en dispositifs carrés dans lesquels ils étaient placés à différentes distances l'un de l'autre. Chaque dispositif est désigné dans la suite par un code, qui comporte le diamètre du piège en centimètres, suivi de la distance entre pièges en mètres. C'est ainsi que nous avions les dispositifs $10-4,10-6,15-4$ et $15-6$, qui comportaient chacun 36 pièges, le dispositif 10-2, qui comportait 64 pièges et le $15-8$, formé de 25 pièges. La dimension et la position relative des différents dispositifs sont précisés sur la figure 1. 


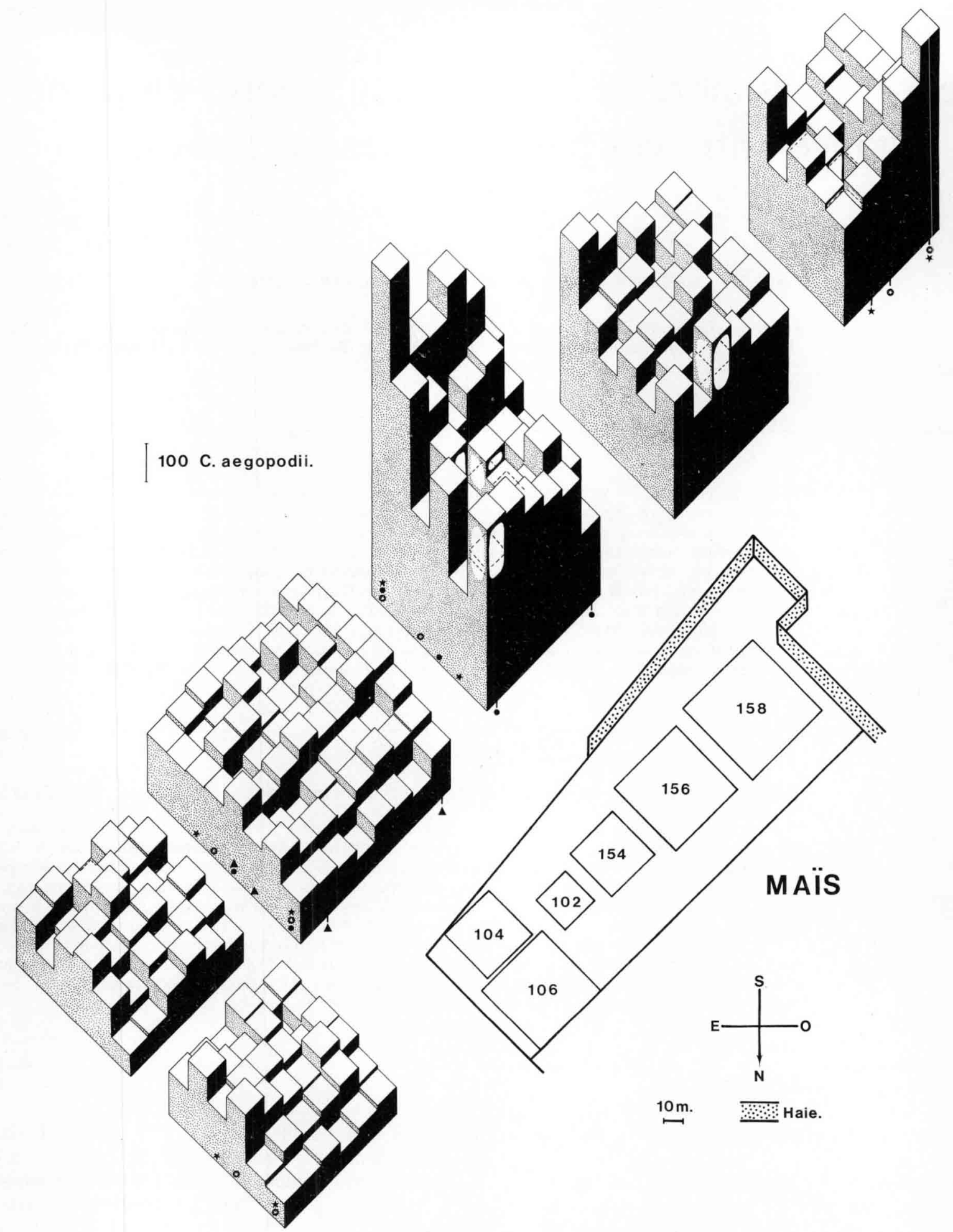

Figure 1

Répartition spatiale des captures de Cavariella aegopodii dans les différents pièges disposés dans le champ de carottes.

La position et la taille relative des différents dispositifs sont précisées sur le plan.

Dans chaque dispositif dont les captures ne sont pas homogènes (cf. tabl. 1), les lignes (ou les colonnes) entre lesquelles existe une différence significative sont signalées par le même symbole.

Distribution of $\mathrm{C}$. aegopodii catches in the traps over the experimental area.

The position and relative size of the different sets of traps are indicated on the plan.

In each set of traps where the catches are not homogeneous ( $c f .:$ table 1), the lines (or the columns) significantly different from each other are pointed out with the same figure.

L'essai a eu lieu en pleine période de vol du puceron de la carotte, Cavariella aegopodii Scop., dont nous étudierons plus particulièrement les captures. Au cours de cette période, 49814 C. aegopodii ont été piégés, ainsi que 3534 «autres aphides» représentant 53 espèces. Les données élémentaires relatives aux captures ne peuvent être transcrites faute de place; elles sont disponibles auprès des auteurs. 


\section{RÉSULTATS}

\section{A. Homogénéité des captures à l'intérieur des dispositifs de piégeage}

La première phase de l'étude des résultats fournis par un dispositif de piégeage consiste à s'intéresser à l'homogénéité des données. Nos résultats ont été obtenus dans un dispositif de type blocs, ce qui nous permet de nous poser dans de bonnes conditions le problème de leur homogénéité. Il s'agit d'échantillons non indépendants et, à défaut de tests mieux adaptés, nous avons effectué des tests non paramétriques de FRIEDMAN de comparaison de plusieurs populations, qui ne préjugent pas de la distribution du nombre de captures par piège. Ces tests ont été effectués d'une part sur les lignes, d'autre part sur les colonnes de chacun des dispositifs. Les résultats sont indiqués dans le tableau 1.

\section{TABLEAU 1}

Résultats des tests de FRIEDMAN de comparaison des captures de Cavariella aegopodii entre les lignes d'une part et les colonnes d'autre part, des différents dispositifs de piégeage.

Results of FRIEDMAN tests comparing catches of C. aegopodii between lines or columns of the various sets of traps.

\begin{tabular}{|c|c|c|c|c|}
\hline Dispositif & Colonne/Ligne & $\chi^{2}$ calculé & $x^{2} 0,05$ & $\chi^{2} 0,01$ \\
\hline \multirow{2}{*}{$10-2$} & $\mathrm{C}$ & $32,3 * *$ & \multirow{2}{*}{14,1} & \multirow{2}{*}{18,5} \\
\hline & $\mathrm{L}$ & $14,6^{*}$ & & \\
\hline \multirow{2}{*}{$10-4$} & C & 9,62 & \multirow{2}{*}{11,1} & \multirow{2}{*}{15,1} \\
\hline & L & 6,45 & & \\
\hline \multirow{2}{*}{$10-6$} & $\mathrm{C}$ & $17,52 * *$ & \multirow[b]{2}{*}{11,1} & \multirow[b]{2}{*}{15,1} \\
\hline & & 4,79 & & \\
\hline \multirow[b]{2}{*}{$15-4$} & $\mathrm{C}$ & $21,62 * *$ & \multirow[b]{2}{*}{11,1} & \multirow[b]{2}{*}{15,1} \\
\hline & $\mathrm{L}$ & & & \\
\hline \multirow[b]{2}{*}{$15-6$} & $\mathrm{C}$ & 8,67 & \multirow[b]{2}{*}{11,1} & \multirow[b]{2}{*}{15,1} \\
\hline & L & 1,91 & & \\
\hline \multirow[b]{2}{*}{$15-8$} & $\mathrm{C}$ & 7,56 & \multirow[b]{2}{*}{9,5} & \multirow[b]{2}{*}{13,3} \\
\hline & $\mathrm{L}$ & $13,12 *$ & & \\
\hline
\end{tabular}

* significatif 0,05

** significatif 0,01

N.B. : Pour se reférer à la figure 1, les lignes sont parallèles à la direction N.E.-S.O.

N.B. : For comparison with figure 1, the lines are parallel with the N.E.-S.W. axis.

Lorsque l'hypothèse d'homogénéité était rejetée, nous avons procédé à la comparaison multiple entre les lignes ou entre les colonnes pour déterminer quels couples présentaient des différences significatives ; ces couples sont signalés sur la figure 1.

Seulement 2 des dispositifs (10-4 et 15-6) se révèlent statistiquement homogènes. A l'observation de la figure 1, il est évident que les pièges de 10 et ceux de $15 \mathrm{~cm}$, en raison de leurs situations dans le champ et peut être de leur différence de taille, se comportent différemment :
- les pièges de $15 \mathrm{~cm}$ capturent d'autant plus de $C$. aegopodii, que l'on est plus près de la haie de $10 \mathrm{~m}$ de haut qui entoure le côté sud de la parcelle; il y a un véritable gradient de captures, celles-ci diminuant à partir de 3 des côtés du dispositif 15 vers le $4^{c}$, c'est-à-dire vers la parcelle de maïs ;

- les dispositifs 10-2 et 10-6, constitués de pièges de $10 \mathrm{~cm}$, présentent 1 ligne de faible capture en bordure côté maïs et 2 lignes plus fortes au milieu, ces différences étant significatives. Les différences entre pièges sont moins accusées pour les pièges de $10 \mathrm{~cm}$ que pour ceux de $15 \mathrm{~cm}$.

Si l'on considère maintenant les captures des « autres pucerons » globalement (résultats des tests non transcrits), seul le dispositif 15-4 présente une hétérogénéité entre ses lignes $\left(\chi_{\text {obs }}^{2}=18,6 ; \chi_{0,01}^{2}=15,1\right)$, la $6^{\mathrm{e}}$ ligne étant significativement différente de la $4^{\mathrm{e}}$, comme cela était également le cas en ce qui concerne les captures de $C$. aegopodii.

Nous ne ferons pas ici d'hypothèse sur les causes des différences de comportement ou des différences d'abondance des aphides au niveau des différents pièges d'un même dispositif, cette question ayant été approfondie par ailleurs (ROBERT et al., 1976). Nous nous bornerons à constater que nous avons pu observer des hétérogénéités statistiquement prouvées dans ces dispositifs, apparemment en relation (dispositifs 15) ou sans relation (dispositifs 10) avec l'environnement.

Notons enfin que, étant donné les remarques ci-dessus sur la différence de situation entre les pièges de 10 et ceux de $15 \mathrm{~cm}$, nous ne comparerons pas les captures de ces 2 types de pièges entre elles, l'incidence de la taille du piègc ayant été d'ailleurs traitée précédemment (RABASSE et al., 1976; BRUNEL \& RABASSE, 1975).

\section{B. Nombre de pièges et précision du résultat}

Une question essentielle lorsque l'on met en place un dispositif de piégeage est de savoir quel est le nombre de pièges nécessaires pour obtenir un résultat suffisamment affiné. Nous avons vu dans le paragraphe précédent que les captures à l'intérieur des dispositifs étaient hétérogènes. En toute rigueur, il n'est donc pas possible de considérer que ces captures, dans leur ensemble, répondent à une même loi de distribution ; il n'est pas possible non plus de séparer de l'ensemble les pièges qui, à l'analyse, se sont révélés être différents des autres. Par ailleurs, pour une première approche de la relation entre taille de l'échantillon et précision du résultat, il est commode de travailler sur des distributions assimilables à des lois normales. Nous avons donc testé la normalité des distributions des nombres de C. aegopodii capturés dans les pièges de chaque dispositif, par les coefficients, $\beta_{1}, \beta_{2}$ de PEARSON et a de GEARY (MORICE, 1972). Au seuil 2 p. 100 de risque, seule la distribution des pucerons dans les pièges du dispositif $15-4$ est significativement différente d'une loi normale au vu du coefficient de symétrie $\beta_{1} ; \beta_{2}$ et a, qui caractérisent l'aplatissement des courbes, ne prennent pas de valeurs significatives (tabl. 2). Si nous choisissons un autre seuil de risque, 5 p. 100 , d'autres distributions, notamment celles des dispositifs composés de pièges de $15 \mathrm{~cm}$ de diamètre, ne pourraient pas être assimilées à des distributions normales.

Dans une population distribuée normalement (DAGNELIE, 1970), le nombre d'échantillons $n$ nécessaires pour obtenir dans 95 p. 100 des cas une erreur maximale L est :

$\mathrm{n}=\frac{(1,960)^{2} \sigma^{2}}{\mathrm{~L}^{2}}$

$\sigma^{2}=$ variance de la population 
TABLEAU 2

Nombres moyens de Cavariclla aegopodii capturés dans les pièges des différents dispositifs et paramètres des distributions de captures. Mean numbers of $\mathrm{C}$. aegopodii caught in the traps of the different sets and parameters of catch distributions.

\begin{tabular}{|c|c|c|c|c|c|c|c|c|}
\hline \multirow{2}{*}{ Dispositif } & \multirow{2}{*}{$\begin{array}{c}\text { Nombre } \\
\text { de } \\
\text { pièges }\end{array}$} & \multicolumn{6}{|c|}{ Paramètres des distributions de captures } & \multirow{2}{*}{$\begin{array}{c}\text { Captures } / \mathrm{m}^{2} \\
\text { de } \\
\text { dispositif }\end{array}$} \\
\hline & & $\bar{x}$ & $\mathrm{~S}_{\mathrm{x}}$ & $\mathrm{c} \%$ & $\beta_{1}$ & $\beta_{2}$ & a & \\
\hline $10-2$ & 64 & 147,9 & 35,0 & 23,7 & $-0,41$ & 2,79 & 0,79 & 37,0 \\
\hline $10-4$ & 36 & 122,6 & 36,1 & 29,4 & 0,09 & 2,48 & 0,80 & 7,7 \\
\hline $10-6$ & 36 & 109,1 & 36,1 & 33,1 & 0,37 & 2,77 & 0,81 & 3,0 \\
\hline $15-4$ & 36 & 423,4 & 142,5 & 33,7 & $1,04 *$ & 4,00 & 0,77 & 26,5 \\
\hline $15-6$ & 33 & 279,5 & 53,9 & 19,3 & 0,78 & 4,53 & 0,73 & 7,8 \\
\hline $15-8$ & 25 & 289,3 & 78,3 & 27,1 & 0,88 & 4,49 & 0,75 & 4,5 \\
\hline
\end{tabular}

$c=$ coefficient de variation, $\beta_{1}=$ coefficient de symétrie, $\beta_{2}=$ coefficient d'aplatissement de PEARSON. a $=$ coefficient de GeARY, * = significativement différent d'une loi normale au seuil de risque 0,02 .

$c=$ coefficient of variation, $\beta_{1}=$ coefficient of skewness, $\beta_{2}=$ PEARSON's coefficient of kurtosis, a = GEARY's coefficient, * $=$ Significantly different from a normal distribution at a 0.02 confidence level.

soit, avec $\mathrm{p}=\frac{\mathrm{L}}{\mathrm{m}} \mathrm{et} \mathrm{c}=\frac{\sigma}{\mathrm{m}}$ :

$\mathrm{p}=\frac{1,960 \mathrm{c}}{\sqrt{\mathrm{n}}}$

$\mathrm{m}=$ moyenne de la population; $\mathrm{c}=$ coefficient de variation de la population; $\mathrm{p}=$ erreur tolérable, exprimée en pourcentage de la moyenne.

Dans nos essais, le coefficient de variation des captures est compris entre 19 et 34 p. 100 (tabl. 2). Il ne semble pas très différent pour les 2 tailles de pièges, tandis que le nombre de captures passe du simple au double et augmente fortement le temps de dépouillement, donc son coût. Cette constatation, si elle se confirmait, semblerait nettement en faveur de l'utilisation de pièges de petite taille (RABASSE et al., 1976). Il est probable que l'on puissc obtenir des coefficients plus bas en choisissant des sites très homogènes et en ne tenant pas compte des rangées de pièges en bordure du dispositif. Nous avons représenté sur la figure 2 l'évolution de l'erreur tolérable, calculée à l'aide de la formule $\left({ }^{2}\right)$, en fonction du nombre de pièges, pour 4 coefficients de variation compris dans la gamme de ceux que nous avons observés. Nous ferons deux remarques sur cette courbe. Une quinzaine de petits pièges de 10 ou $15 \mathrm{~cm}$ de diamètre permettent d'obtenir, dans 95 p. 100 des cas, une très bonne précision, de l'ordre de 10 à 20 p. 100 des captures. D'autre part, 5 pièges donnent une précision de 30 p. 100 au moins. Les vols d'aphides (ROBERT \& ROUZE-JOUAN, 1976) et en particulier de C. aegopodii (BRUNEL \& RABASSE, 1977) se manifestent par des variations brusques des captures d'un coefficient 10 . On peut donc penser qu'il suffit d'un petit nombre de pièges jaunes pour traduire avec une certaine précision l'évolution de ces captures.

\section{Influence de la distance entre pièges}

Un des objectifs de cet essai était de préciser l'importance de la distance entre pièges. Les valeurs moyennes des captures sont indiquées dans le tableau 2. Nous avons

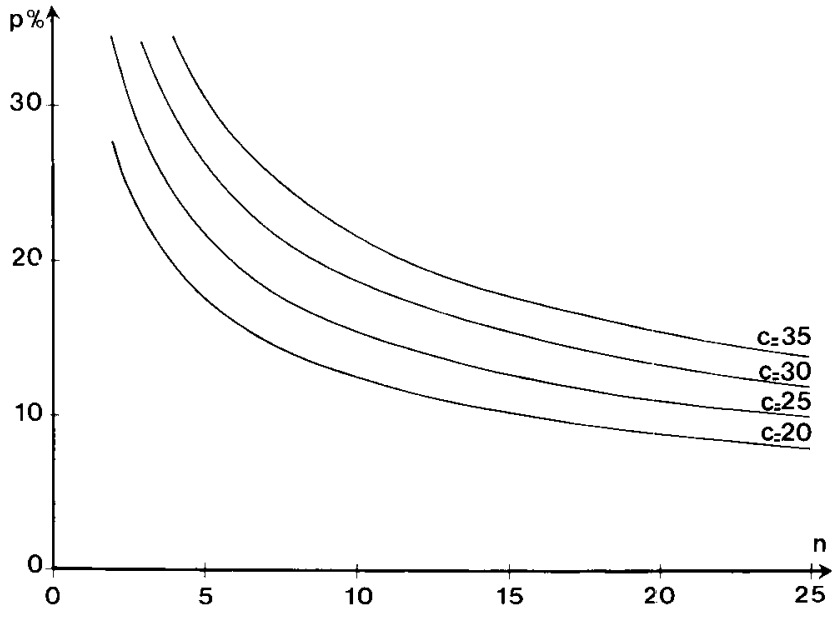

Figure 2

Relation théorique entre la précision de l'estimation du nombre de captures $(p)$ et le nombre de pièges $(n)$ pour différents coefficients de variation (c) caractérisant la distribution du nombre d'aphides par piège.

Theoretical relationship between precision in the estimation of number of catches $(p)$ and number of traps $(n)$ for various coefficients of variation (c) of the distribution of the number of aphids per trap.

comparé les piégeages dans les différents dispositifs pris 2 à 2 dans l'ordre de taille croissante. Chaque comparaison a donné lieu à un test de WILCOXON, test de rangs appliqué à 2 échantillons indépendants (tabl. 3 ).

Pour chaque taille de pièges, les captures sont significativement différentes entre le dispositif à pièges rapprochés et le dispositif moyen, mais pas entre ce dernier et le dispositif à pièges éloignés. On sait que, dans un dispositif de pièges jaunes, lorsque l'on rapproche ces pièges le nombre de captures par piège augmente puis tend à plafonner par suite de «l'épuisement» du milieu (RoTH, 1971; FINCH \& SKInNER, 1974). Nous n'avons observé que 3 distances pour chaque type de piège, cependant il semble que les captures 


\section{TABLEAU 3}

Résultats des tests de WILCoxon de comparaison des captures de Cavariclla aegopodii dans les différents dispositifs de piégeage pris deux à deux.

Results of WILCOXON tests comparing catches of $\mathrm{C}$. aegopodii in the various sets of traps arranged two by two.

\begin{tabular}{cc}
\hline \hline Comparaison & u observé \\
\hline $10-2 / 10-4$ & $3,48^{* *}$ \\
\hline $10-4 / 10-6$ & 1,54 \\
\hline $15-4 / 15-6$ & $4,97^{* *}$ \\
\hline $15-6 / 15-8$ & 0,38 \\
\hline $\mathrm{u}_{0,975}=1,96\left({ }^{*}\right)$ & $\mathrm{u}_{0.4 \% 5}=2,58(* *)$ \\
\hline \hline
\end{tabular}

se stabilisent ici lorsqu'on éloigne les pièges dans les 2 essais. Nous sommes en pleine période de vol du puceron de la carotte et la densité des pièges est bien plus faible que celle qui peut permettre un épuisement des aphides (environ $5000 \mathrm{C}$. aegopodii et 380 "autres aphides" piégés/ha/jour). D'ailleurs, le calcul du nombre de pucerons de la carotte capturés par unité de surface de dispositif (tabl. 2) montre bien une augmentation régulière des captures lorsque les pièges sont plus proches les uns des autres. Par contre, on peut penser qu'à l'écartement moyen ou grand, les pièges se comportent comme des pièges isolés. Au plus petit écartement, à l'effet «attraction du piège " s'ajouterait un effet «attraction du dispositif » qui augmenterait l'effectif piégé.

Notons que pour les « autres aphides», nous n'avons trouvé de différence significative qu'entre les résultats des dispositifs $10-2$ et $10-4\left(\mathrm{U}_{\text {obs. }}=2,67\right)$.

\section{Influence des différents paramètres sur l'indice de diversité des captures}

Le tableau 4 précise le nombre d'espèces et d'individus capturés. Les aphides récoltés ont été triés en 53 unités taxonomiques; dans la plupart des cas, l'espèce a été identifiée ; dans de rares cas cependant, ces unités sont hétérogènes (groupe "Aphis fabae", Hyperomyzus $s p p . .$.$) . Ces chiffres ne tiennent volontairement pas compte$ de $C$. aegopodii, espèce très dominante et présente partout.
Les indices de diversité calculés diminuent quand l'écartement des pièges augmente. Entre les deux plus grandes distances, les indices de WILLIAMS ne sont pas significativement différents (pièges de $10 \mathrm{~cm}$ ) ou sont à peine différents (pièges de $15 \mathrm{~cm}$ ) ; par contre, entre la plus faible distance et les 2 autres, les indices sont très différents. Ici encore, nous sommes amenés à conclure à un "effet dispositif", dont nous avons recherché la cause de deux façons, mais sans pouvoir aboutir :

- en cherchant quelles espèces sont capturées seulement dans certains dispositifs. Ces espèces ne sont représentées que par un seul individu, ce qui ne permet pas de conclure ;

- en cherchant si l'abondance relative d'une espèce (rang d'abondance) diffère selon les dispositifs. Il y a dans l'ensemble une bonne homogénéité.

Dans un travail précédent (RABASSE et al., 1976), nous avions constaté une forte variation de l'indice de diversité de Williams, aussi bien que de celui de SHANNON, avec la dimension des pièges jaunes. Dans cette expérimentation, le nombre de pièges de chaque taille étant constant, la taille de l'échantillon variait donc avec la dimension des pièges. Le présent essai nous permet de vérifier l'indépendance de l'indice de diversité de WiLliams par rapport à la taille de l'échantillon. Pour cela, nous avons rassemblé les captures des pièges par groupes de 9,18 et 36 pièges tirés au hasard (dispositifs $10-4,10-6,15-4$ et $15-6$ ), ou par $4,8,16,32$ et 64 (dispositif 10-2). La figure 3 montre les valeurs des nombres d'espèces et d'individus obtenus après chacun de ces regroupements, ainsi que 3 courbes sur lesquelles l'indice de diversité de WiLliams conserve la même valeur. Il est clair que nous ne retrouvons pas le phénomène évoqué ci-dessus, alors que les effectifs sont comparables et que, dans cet essai, l'indice de diversité est indépendant de la taille de l'échantillon. Cette constatation nous amène à la conclusion que si l'indice de diversité des captures augmente avec la taille des pièges, c'est que plus les pièges sont grands, plus la biocénose prospectée est variée. La hauteur à laquelle des espèces ayant des comportements de vol différents sont susceptibles d'être attirées est peut-être un des facteurs à prendre en considération à cet égard.

\section{DISCUSSION - CONCLUSION}

Nous avons étudié dans ce travail les captures de petits pièges jaunes formant des dispositifs de grandes dimensions en zone non ouverte.

Indépendamment de l'effet de l'environnement, nous

TABLEAU 4

Nombres d'espèces ( $S$ ) et d'individus (N) d'aphides autres que Cavariella aegopodii capturés dans chacun des dispositifs de piégeage et indice de diversité de SHANNON (Ish.) et de WILLIAMS $(\alpha)$.

Numbers of species (S) and of individuals $(N)$ of aphids, C. aegopodii excepted, caught in each set of traps and diversity indices of SHANNON (Ish.) and of WILLIAMS $(\alpha)$.

\begin{tabular}{cllllll}
\hline \hline Dispositif & $10-2$ & $10-4$ & $10-6$ & $15-4$ & $15-6$ & $15-8$ \\
\hline $\mathrm{N}$ & 651 & 284 & 300 & 875 & 787 & 637 \\
\hline $\mathrm{S}$ & 36 & 26 & 27 & 42 & 31 & 32 \\
\hline Ish. & 3,74 & 3,75 & 3,48 & 3,68 & 3,24 & 3,19 \\
\hline$\alpha$ & $8,21 \pm 0,69$ & $6,97 \pm 0,78$ & $7,19 \pm 0,78$ & $9,20 \pm 0,70$ & $6,44 \pm 0,55$ & $7,10 \pm 0,62$ \\
\hline \hline
\end{tabular}




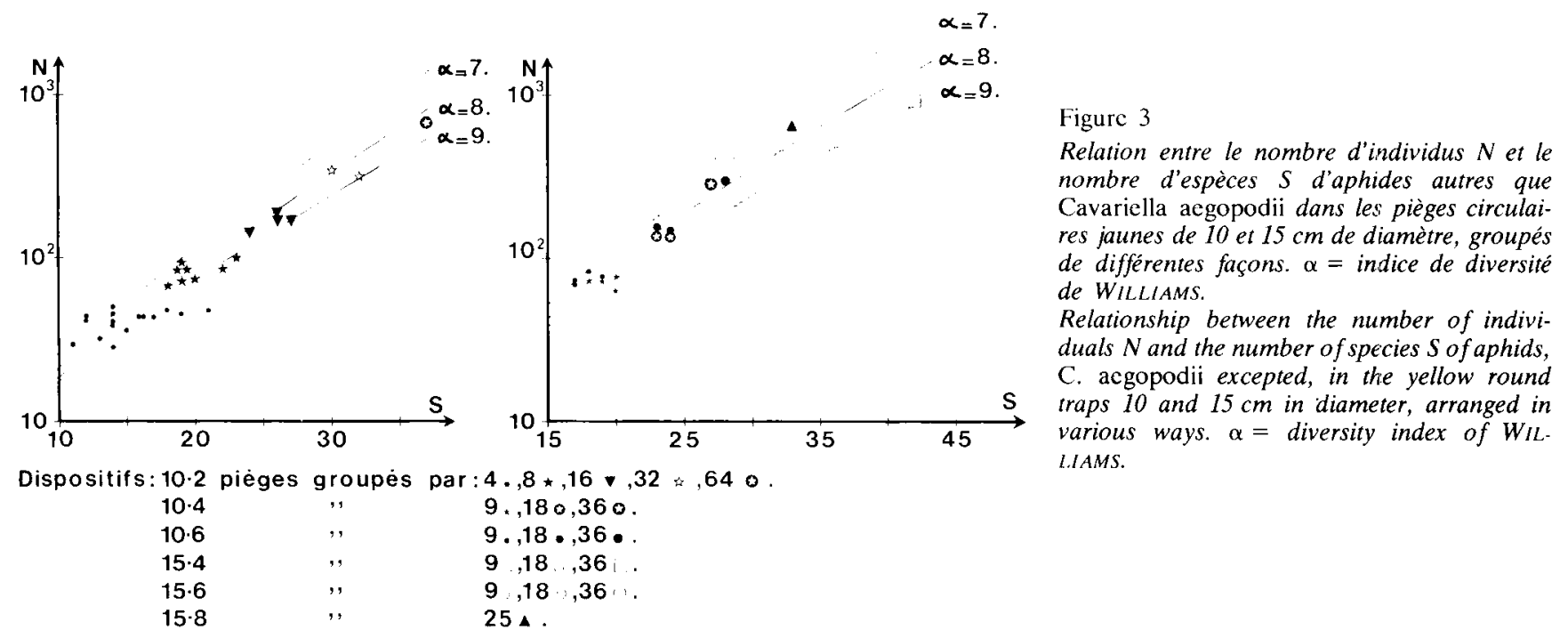

avons trouvé qu'à «l'effet piège » sc superpose un «effet dispositif de piégeage », qui se manifeste de 3 façons :

- en modifiant les captures de pièges ayant une localisation particulic̀re (rangs de bordure, rangs du milieu des dispositifs formés de pièges de $10 \mathrm{~cm}$ ).

- en augmentant les captures de $C$. aegopodii par piège lorsque les pièges sont rapprochés, formant un dispositif plus dense.

- en augmentant la diversité des captures de pucerons dans ces mêmes dispositifs à pièges rapprochés.

La mise en évidence d'un effet dispositif implique que l'on en tienne compte lorsque, comme par exemple Wolf et al. (1971), l'on essaie de reconstituer par le calcul l'action de différentes combinaisons de pièges. D'un point de vue pratique, elle conduit surtout de la notion du piège standard à celle de dispositif de piégeage standard. Enfin, elle appelle des expérimentations complémentaires pour comprendre ce que représente le piège isolé ou la ligne de pièges par rapport au piège inséré dans un dispositif.

Peu d'auteurs se sont attachés à étudier le nombre de pièges nécessaires pour obtenir un résultat suffisamment précis. OBRTEL (1971) a effectué une telle étude pour les pièges de BARBER utilisés pour les coléoptères. En ce qui concerne les aphides, PELET et al. (1954) avaient posé le problème de façon théorique. De ce point de vue, nos résultats sont plutôt encourageants, montrant qu'un petit nombre de pièges peut suffire pour une précision satisfaisante, au moins en ce qui concerne les aphides dont les captures sont l'objet de fluctuations importantes. Ils suggèrent également que pour un même travail d'identification, pour un même nombre d'insectes recueillis, l'utilisation de petits pièges pourrait fournir un résultat plus précis ; ici encore une expérimentation complémentaire serait nécessaire.

Enfin en ce qui concerne l'image d'ensemble de la population aphidienne capturée dans nos conditions expérimentales, l'indice de diversité se précise bien entendu avec le nombre de pièges et le nombre d'insectes capturés ; il est affecté par le type de dispositif, comme il l'était par la dimension des pièges, mais pas par la taille de l'échantillon recueilli.

\section{RÉFÉRENCES BIBLIOGRAPHIQUES}

Brunel E., Langouet L., 1970. Influence des caractéristiques optiques du milieu sur les adultes de Psila rosae Fabr. (Diptères, Psilides): attractivité de surfaces colorées, rythme journalier d'activité. C.R. Soc. Biol., 164, 1638-1644.

Brunel E., Lefeuvre J. C., Missonnier J., Robert Y., Trehen P., 1980. La faune du bocage. Incidences de l'arasement des talus boisés. Conséquences agricoles. Bull. tech. Inf. Min. Agric., 354355, $725-767$.

Brunel E., Rabasse J. M., 1975. Influence de la forme et de la dimension de pièges à eau colorés en jaune sur les captures d'insectes dans une culture de carottc. Cas particulier des Diptères. Ann. Zool. Ecol. anim., 7, 345-364.

Brunel E., Rabasse J.-M., 1977. Cavariella aegopodii Scop. (Hom. Aphididae) en culture de carotte dans l'Ouest de la France. I. Evolution des populations. Ann. Zool. Ecol. anim., 9, 469-480.

Dagnelie P., 1970. Théorie et méthodes statistiques, 2, 18-20, Duculot, Gembloux.
Finch S., Skinner G., 1974. Some factors affecting the efficiency of water-traps for capturing cabbage root flies. Ann. appl. Biol., 77, 213-226.

Morice E., 1972. Tests de normalité d'une distribution observée. Rev. Stat. appl., 20, 5-35.

Obrtel R., 1971. Number of pitfall traps in relation to the structure of the catch of soil surface Coleoptera. Acta entomol. bohemoslov, 68, 300-309.

Pelet F., Munster J., Bovey R., 1954. Essai de contrôle du vol du puceron Myzus persicae Sulz. à l'aide de pièges jaunes. Landwirtsch. Jahrb. Schweiz., 55, 917-930.

Rabasse J. M., Brunel E., Delecolle R., Rouze-Jouan J., 1976. Influence de la dimension de pièges à eau colorés en jaune sur les captures d'aphides dans une culture de carotte. Ann. Zool. Ecol. anim., 8, 39-52.

Robert Y., Brunel E., Malet P., Bautrais P., 1976. Distribution spatiale de pucerons ailés et de diptères dans une parcelle de bocage, en fonction des modifications climatiques provoquées par les haies. C.R. Table ronde C.N.R.S. "Ecosystèmes bocagers", Rennes, 1976, 427-435. 
Robert Y., Rabasse J. M., Rouze-Jouan J., 1974. Sur l'utilisation des pièges jaunes pour la capture des pucerons en culture de pomme-de-terre. I. Influence de la hauteur de piégeage. Ann. Zool. Ecol. anim., 6, 349-372.

Robert Y., Rouze-Jouan J., 1976. Activité saisonnière de vol des pucerons (Hom., Aphididae) dans l'Ouest de la France. Résultats de neuf années de piégeage (1967-1975). Ann. Soc. entomol. Fr. (N.S.), 12, 671-690.
Roth M., 1971. Contribution à l'étude éthologique du peuplement d'insectes d'un milicu herbacé. Mémoire O.R.S.T.O.M., 53, $118 \mathrm{pp}$.

Wolf W. W., Kishaba A. N., Toba H. H., 1971. Proposed method for determining density of traps required to reducc an insect population. J. econ. Entomol., 64, 872-877. 